\title{
Relationships between braid length and the number of braid strands
}

\author{
CORnelia A VAN COTT
}

\begin{abstract}
For a knot $K$, let $\ell(K, n)$ be the minimum length of an $n$-stranded braid representative of $K$. Fixing a knot $K, \ell(K, n)$ can be viewed as a function of $n$, which we denote by $\ell_{K}(n)$. Examples of knots exist for which $\ell_{K}(n)$ is a nonincreasing function. We investigate the behavior of $\ell_{K}(n)$, developing bounds on the function in terms of the genus of $K$. The bounds lead to the conclusion that for any knot $K$ the function $\ell_{K}(n)$ is eventually stable. We study the stable behavior of $\ell_{K}(n)$, with stronger results for homogeneous knots. For knots of nine or fewer crossings, we show that $\ell_{K}(n)$ is stable on all of its domain and determine the function completely.
\end{abstract}

57M25; 20F36

\section{Introduction}

The first of many connections between knot theory and braid theory was discovered in 1923 when Alexander proved that every oriented knot or link is isotopic to a closed braid [1]. The complicating twist is that this braid is not unique. In fact, every knot is isotopic to infinitely many distinct closed braids.

Many efforts have been made to study knots using their braid representatives. In particular, braid theory is used to define two interesting knot invariants:

Definition 1 The braid index of a knot $K, \operatorname{br}(K)$, is the minimum number of strands needed to express $K$ as a closed braid.

Definition 2 The braid length of a knot $K, \ell(K)$, is the minimum number of crossings needed to express $K$ as a closed braid.

Consider the second knot invariant defined above: braid length. One might conjecture that a braid with the minimum number of crossings also has the minimum number of strands. Thomas Gittings computed braid lengths for a large number of knots, and in the midst of these computations he encountered counterexamples to this conjecture [5]. The first counterexample is the knot $10_{136}$. This knot has braid index $\operatorname{br}\left(10_{136}\right)=4$. 
Gittings found that all 4 -braids with closure $10_{136}$ have at least 11 crossings. However, he found 5-braids with closure $10_{136}$ with only 10 crossings (see Figure 1). The fact that adding strands might actually decrease the number of necessary crossings is unexpected, and in this paper we will investigate how the number of braid strands constrains or determines the length of braids associated to a knot $K$. To this end, we make the following definition:

Definition $3 \quad \ell(K, n)=$ minimum number of crossings needed to express $K$ as a closed $n$-braid, where $n \geq \operatorname{br}(K)$.

Observe that this newly defined class of invariants is related to the braid length $\ell(K)$ as follows:

$$
\ell(K)=\min _{n \geq \operatorname{br}(K)}\{\ell(K, n)\}
$$

Fixing a knot $K$, we can consider $\ell(K, n)$ to be a function from a subset of the integers to the positive integers, which we denote by $\ell_{K}(n)$ :

$$
\ell_{K}: \mathbf{N}_{\geq \operatorname{br}(K)} \longrightarrow \mathbf{N}
$$

From our previous discussion, we already know a few values of the function $\ell_{10_{136}}(n)$ :

$$
\begin{aligned}
& \ell_{10_{136}}(4)=11 \\
& \ell_{10_{136}}(5)=10
\end{aligned}
$$

Theorem 5 will show that $\ell_{10_{136}}(n)=n+5$ for $n \geq 5$, so the function drops one time from $\ell_{10_{136}}(4)$ to $\ell_{10_{136}}(5)$ and then increases monotonically thereafter. Knots similar to $10_{136}$ whose associated functions decrease in value at some point are interesting, for these are precisely the knots for which increasing the number of strands actually decreases the number of necessary crossings.

We begin in Section 2 of this paper by establishing some general results about the behavior of $\ell_{K}(n)$.

Theorem 4 Let $K$ be a knot. The function $\ell_{K}(n)$ has the following properties:

(1) $\ell_{K}(n+1) \leq \ell_{K}(n)+1$ for all $n \geq \operatorname{br}(K)$.

(2) $\ell_{K}(n)-n \equiv 1(\bmod 2)$.

(3) $\ell_{K}(n) \geq n+2 g(K)-1$, where $g(K)$ denotes the genus of $K$.

From parts 1 and 3 of this theorem, it follows that every function $\ell_{K}(n)$ is eventually stable. That is, for every knot $K$ there exist positive integers $a$ and $n_{0}$ (depending on $K$ ) such that

$$
\ell_{K}(n)=n+a
$$



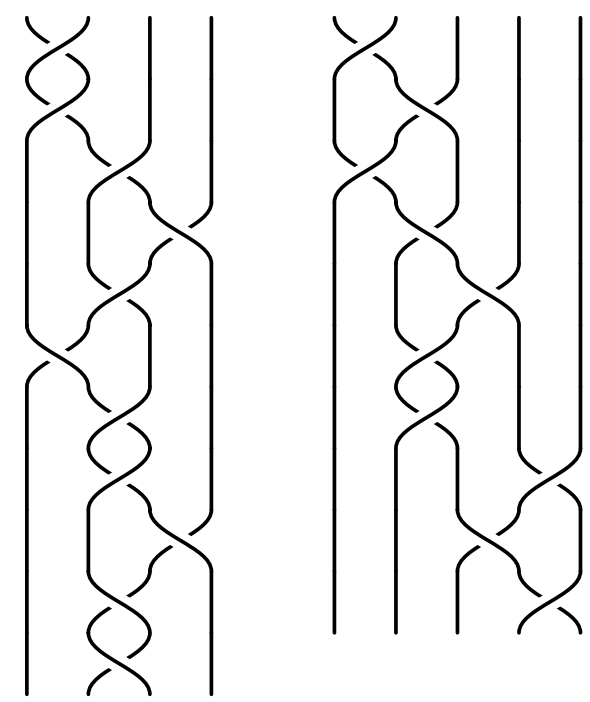

Figure 1: A 4-braid with closure $10_{136}$ and length 11 (left) and a 5-braid with closure $10_{136}$ and length 10 (right)

for all $n \geq n_{0}$. Because of part 2 of the above theorem, $a$ must be odd.

Section 3 investigates the stable behavior of functions $\ell_{K}(n)$. First, we find a point after which the function $\ell_{K}(n)$ is stable, given any functional value.

Theorem 5 Let $K$ be a prime knot. Suppose $\ell_{K}(m)=c$ for some $m$.

(1) If $c \leq 2 m$, then $\ell_{K}(n)$ is stable for all $n \geq m$.

(2) If $c>2 m$, then $\ell_{K}(n)$ is stable for all $n \geq c-m$.

Stronger results arise for specific families of knots. In the case of homogeneous knots (defined below), a point $n_{0}$ after which the function is stable can be determined without computing an initial functional value, and the constant $a$ is determined by the genus of the knot.

Definition 6 A braid word $\beta \in B_{n}$ is homogeneous if each standard braid generator $\sigma_{i}$ appears at least once in $\beta$ and the exponent on $\sigma_{i}$ has the same sign in each appearance in the braid word $\beta$ (thus if $\sigma_{i}$ appears, then $\sigma_{i}^{-1}$ does not appear).

Definition 7 A knot $K$ is a homogeneous knot if there exists a homogeneous braid $\beta \in B_{m}$ for some $m$ with its closure $\widehat{\beta}$ isotopic to $K$. 
Theorem 8 Let $K$ be a homogeneous knot with an $m$-strand homogeneous braid representative $\beta \in B_{m}$. Then $\ell_{K}(n)=n+2 g(K)-1$ for all $n \geq m$.

Since every function $\ell_{K}(n)$ is eventually stable, we can fix an (odd) integer $a$ and find all knots whose stable functions are $\ell_{K}(n)=n+a$. For the special case $a=1$, the collection of knots is particularly limited.

Theorem 9 Let $K$ be a knot. Then $\ell_{K}(n)=n+1$ for some $n$ if and only if $K$ is the trefoil or the figure eight knot. Hence, $\ell_{K}(n) \geq n+3$ for all nontrivial knots $K$ which are not the trefoil or figure eight.

More generally, we have the following result.

Theorem 10 Let $a$ be an odd integer. There are only finitely many prime knots $K$ with stable function $\ell_{K}(n)=n+a$.

Next, we investigate conditions under which $\ell_{K}(n)$ is stable on its entire domain.

Theorem 11 Let $K$ be a knot. Let $\beta \in B_{\mathrm{br}(K)}$ be a braid of length $\ell(K)$ with closure $K$, where $\operatorname{br}(K)$ is the braid index of $K$.

(1) If $\ell(K)<2 \operatorname{br}(K)+2$, then $\ell_{K}(n)$ is stable on its entire domain.

(2) If $\ell(K)=\operatorname{br}(K)+2 g(K)-1$, then $\ell_{K}(n)$ is stable on its entire domain.

In Section 4, we use this theorem along with some further work to determine $\ell_{K}(n)$ for all knots $K$ with nine or fewer crossings.

Theorem 12 Let $K$ be a knot with nine or fewer crossings. Then $\ell_{K}(n)$ is stable on its entire domain.

For background information about braids, see Birman [2], Birman and Brendle [3] and Murasugi and Kurpita [9]. Except where otherwise noted, all knot enumeration and information is taken from Cha and Livingston [4].

Acknowledgments I thank Chuck Livingston for his assistance. Thanks also to Mark Kidwell and the referee for their valuable comments and suggestions. Jiho Kim wrote the computer program mentioned in Section 4.

Algebraic 83 Geometric Topology, Volume 7 (2007) 


\section{Properties of $\ell_{K}(n)$}

A priori, there seem to be few restrictions on the behavior of the function $\ell_{K}(n)$. In this section we will establish some general properties of $\ell_{K}(n)$ which show that we actually are working in a rather structured environment. For example, the parity restriction of part 2 of Theorem 4 rules out the possibility that $\ell_{K}(n)=\ell_{K}(n+1)$ for some $n$.

Theorem 4 Let $K$ be a knot. The function $\ell_{K}(n)$ has the following properties:

(1) $\ell_{K}(n+1) \leq \ell_{K}(n)+1$ for all $n \geq \operatorname{br}(K)$.

(2) $\ell_{K}(n)-n \equiv 1(\bmod 2)$.

(3) $\ell_{K}(n) \geq n+2 g(K)-1$, where $g(K)$ denotes the genus of the knot $K$.

Proof Given a braid $\beta \in B_{n}$ with closure $K$, the stabilized braid $\beta \sigma_{n} \in B_{n+1}$ also has closure $K$. Therefore,

$$
\ell_{K}(n+1) \leq \ell_{K}(n)+1 .
$$

Any braid $\beta$ whose closure is $K$ determines a Seifert surface $S_{\beta}$ for the knot. This surface has $n$ disks and $c$ bands, where $n$ (respectively, $c$ ) is the number of strands (respectively, crossings) of $\beta$. The genus of this surface is

$$
g\left(S_{\beta}\right)=\frac{1+c-n}{2} .
$$

Since the genus of a surface is an integer, it follows that $c-n \equiv 1(\bmod 2)$. Let $\beta \in B_{n}$ be a length minimizing $n$-braid for $K$. Then $c=\ell_{K}(n)$. It follows that $\ell_{K}(n)-n \equiv 1$ $(\bmod 2)$, proving part 2 of the theorem. Moreover, the Seifert surface $S_{\beta}$ satisfies $g(K) \leq g\left(S_{\beta}\right)=\left(1+\ell_{K}(n)-n\right) / 2$. Therefore, $\ell_{K}(n) \geq n+2 g(K)-1$.

We remark here that the lower bound found in part 3 of Theorem 4 is sharp for an infinite collection of knots. Specifically, Theorem 8 will show that for any homogeneous knot $K$,

$$
\ell_{K}(n)=n+2 g(K)-1
$$

for all large $n$. On the other hand, an infinite collection of knots also exists for which $\ell_{K}(n)$ does not reach the lower bound of the above theorem. This follows from Theorem 10.

Combining the upper and lower bounds of Theorem 4,

$$
n+2 g(K)-1 \leq \ell_{K}(n) \leq \ell_{K}(n-1)+1,
$$


it follows that the function $\ell_{K}(n)-n$ is eventually constant. Therefore, for every knot $K$ there exist positive integers $a$ and $n_{0}$ (depending on $K$ ) such that

$$
\ell_{K}(n)=n+a
$$

for all $n \geq n_{0}$. We say that $\ell_{K}(n)$ is stable after the point $n_{0}$ and call the function $n+a$ the stable function of $K$. Observe that by part 2 of the above theorem, $a$ must be an odd number.

\section{Stability of $\ell_{K}(n)$}

Our next step in studying $\ell_{K}(n)$ is understanding the stable behavior of the function. Several questions arise: At what point does the function become stable? What is the value of the function once it is stable? How many knots have the same stable function?

We begin with the first question of finding a point $n_{0}$ after which a function is stable. Theorem 5 gives such a point, given that one can compute any single value of the function.

Theorem 5 Let $K$ be a prime knot. Suppose $\ell_{K}(m)=c$ for some $m$.

(1) If $c \leq 2 m$, then $\ell_{K}(n)$ is stable for all $n \geq m$. In particular,

$$
\ell_{K}(n)=c+n-m \text { for all } n \geq m .
$$

(2) If $c>2 m$, then $\ell_{K}(n)$ is stable for all $n \geq c-m$. In particular,

$$
\ell_{K}(n)=\ell_{K}(c-m)+n-(c-m) \text { for all } n \geq c-m .
$$

Proof of (1) Suppose for contradiction that $\ell_{K}(n) \neq c+n-m$ for some $n>m$. From the upper bound in Theorem 4, the value of $\ell_{K}(n)$ is certainly bounded above by $c+n-m$ (simply take the braid which realizes $\ell_{K}(m)=c$ and stabilize it $n-m$ times). Furthermore, by the parity condition in Theorem 4 , the value of $\ell_{K}(n)$ has the same parity as $c+n-m$. Therefore, $\ell_{K}(n)=c+n-m-2 p$ where $p>0$. Geometrically, this means there is a braid $\beta \in B_{n}$ with closure $K$ and $c+n-m-2 p$ crossings.

We claim that $\beta$ can be destabilized $n-m$ times. To see this, count the number of occurrences of $\sigma_{i}^{ \pm 1}$ in the braid word $\beta$ for each $i \in\{1,2, \ldots, n-1\}$. This gives us an array of $n-1$ integers which sum to $c+n-m-2 p$. For example, the braid $\sigma_{3} \sigma_{2} \sigma_{1} \sigma_{3}^{-1} \sigma_{4} \sigma_{2} \sigma_{5}^{-1} \in B_{6}$ corresponds to the array $(1,2,2,1,1)$. None of the integers in the array associated to $\beta$ are zero (for otherwise $K$ would be a split link).

Algebraic ${ }^{3} \mathcal{G}$ Geometric Topology, Volume 7 (2007) 
Moreover, a careful arithmetic calculation using the pigeonhole principle shows that at least $n-c+m+2 p-2$ of the entries equal 1 .

The existence of a " 1 " in the array implies that $\beta$ is the connected sum of two knots. Since we assumed that $K$ is prime, it must be that one summand is the unknot. So the braid is split by the single crossing into two sides: one side of the braid corresponds to the unknot, and the other corresponds to $K$. Since $\beta$ has a minimal number of crossings, it follows that the part of the braid corresponding to the unknot also has a minimal number of crossings. The unknot can be expressed as a $k$-braid via $\sigma_{1} \sigma_{2} \cdots \sigma_{k-1}$. The integer array corresponding to this braid is $(1,1, \ldots, 1)$, which implies that the braid has minimal length (a smaller length would force one of the integers to be 0 ). Thus the integer array for $\beta$ must be of the form $\left(1, \ldots, 1, x_{1}, x_{2}, \ldots, x_{r}, 1, \ldots, 1\right)$, where none of the $x_{i}$ 's are 1 .

It follows that $\beta$ can be destabilized $n-c+m+2 p-2$ times. In particular, we can destabilize $\beta n-m$ times to obtain an $m$-braid, since

$$
\begin{aligned}
n-c+m+2 p-2 & \geq n-2 m+m+2 p-2 \\
& =n-m+2 p-2 \\
& \geq n-m .
\end{aligned}
$$

The resulting $m$-braid has $c-2 p$ crossings and closure $K$. This contradicts the assumption that $\ell_{K}(m)=c$. So we conclude that $\ell_{K}(n)=c+n-m$ for all $n \geq m$.

Proof of (2) To begin, we show $\ell_{K}(c-m)=2(c-m)-2 t$ for some $t \geq 0$. This follows (as in part 1) from the fact that the braid realizing $\ell_{K}(m)=c$ can be stabilized $c-2 m$ times, yielding a $(c-m)$-braid with $2(c-m)$ crossings. Thus $\ell_{K}(c-m) \leq$ $2(c-m)$. In addition, $\ell_{K}(c-m)$ must have the same parity as $2(c-m)$. Therefore, $\ell_{K}(c-m)=2(c-m)-2 t$ for some $t \geq 0$.

Now we compute $\ell_{K}(n)$ where $n \geq c-m$. Again, we know that

$$
\begin{aligned}
\ell_{K}(n) & \leq \ell_{K}(c-m)+n-(c-m) \\
& =c-m+n-2 t
\end{aligned}
$$

by the upper bound of Theorem 4 . And, taking into account the parity conditions, we know that $\ell_{K}(n)=c-m+n-2 p$, for some $p \geq t \geq 0$. To prove the theorem, we need to show $p=t$. Suppose (for contradiction) that $p>t$. Then there exists a braid $\gamma \in B_{n}$ with closure isotopic to $K$ and $c-m+n-2 p$ crossings, where $p>t \geq 0$.

As in part 1, consider the array of integers associated to the braid $\gamma$ that counts the number of times each braid generator appears. The sum of the $n-1$ numbers in the 
array is $c-m+n-2 p$, and none of the numbers are 0 (for otherwise $K$ would be a split link). The pigeonhole principle implies that the array has at least $n-c+m-2+2 p$ entries equal to 1.

As before, the braid $\gamma$ can be destabilized $n-c+m-2+2 p$ times. In particular, we can destabilize the braid $n-c+m$ times, since $p>0$. The resulting braid, $\gamma^{\prime} \in B_{c-m}$ has length $2(c-m)-2 p$. This is a contradiction to $\ell_{K}(c-m)=2(c-m)-2 t$, established earlier. So we conclude

$$
\begin{aligned}
\ell_{K}(n) & =c-m+n-2 t \\
& =\ell_{K}(c-m)+n-(c-m) .
\end{aligned}
$$

The following example emphasizes how quickly Theorem 5 can resolve the computation of a function.

Example 13 The trefoil $T$ is represented by the 2-braid $\alpha=\sigma_{1}^{3}$. This implies that $\ell_{T}(2) \leq 3$. However, since $T$ is a 3 -crossing knot, strict inequality is impossible, so $\ell_{T}(2)=3$. Now that we have computed a function value of $\ell_{T}(n)$, we can apply Theorem 5 part 1 . It follows that

$$
\ell_{T}(n)=\ell_{T}(2)+n-2=n+1
$$

for all $n \geq 2$. Thus the function $\ell_{T}(n)$ is stable on all of its domain.

One weakness of Theorem 5 is that the hypotheses of the theorem require that a functional value be computed. This may not be an easy task in some cases. In addition, part 2 of the theorem defines the stable function in terms of $\ell_{K}(c-m)$, which may not be known. These difficulties can be avoided if we restrict our attention to a specific family of knots: homogeneous knots (see Definition 7). In this special case, the stable function is determined by the genus of the knot, and the point after which the function is stable no longer depends on a function computation.

Theorem 8 Let $K$ be a homogeneous knot with an $m$-strand homogeneous braid representative $\beta \in B_{m}$. Then $\ell_{K}(n)=n+2 g(K)-1$ for all $n \geq m$.

The proof of this theorem relies on a particular result of Stallings.

Theorem 14 [10] Let $\beta$ be a homogeneous braid. Then $\hat{\beta}$ is a fibered link with fiber surface $S_{\beta}$. 
Since $S_{\beta}$ is a fiber surface for $\hat{\beta}$, it is a minimal genus Seifert surface:

$$
g\left(S_{\beta}\right)=g(\widehat{\beta}) .
$$

With this information in hand, we proceed to the proof of Theorem 8.

Proof of Theorem 8 Suppose $\beta$ has $c$ crossings. Let $S_{\beta}$ denote the Seifert surface (with $m$ disks and $c$ bands attached) corresponding to the closed homogeneous braid $\hat{\beta}$. Applying Theorem 14 and Equation (3-1), we have

$$
g(K)=g\left(S_{\beta}\right)=\frac{1+c-m}{2} .
$$

Rearranging terms, we have $c=m+2 g(K)-1$. This is the lower bound for $\ell_{K}(m)$ found in part 3 of Theorem 4 . Therefore $\ell_{K}(m)=m+2 g(K)-1$. Now by parts 1 and 3 of Theorem 4 , it follows that $\ell_{K}(n)=n+2 g(K)-1$ for all $n \geq m$.

Note that given a homogeneous knot $K$ of braid index $n$, we cannot necessarily find a homogeneous braid in $B_{n}$ whose closure is $K$. In fact, Stoimenow found an infinite set of examples where no such braid exists [11, Theorem 1]. By definition, we are only guaranteed that for some integer $m \geq n$, there is a homogeneous braid in $B_{m}$ whose closure is $K$.

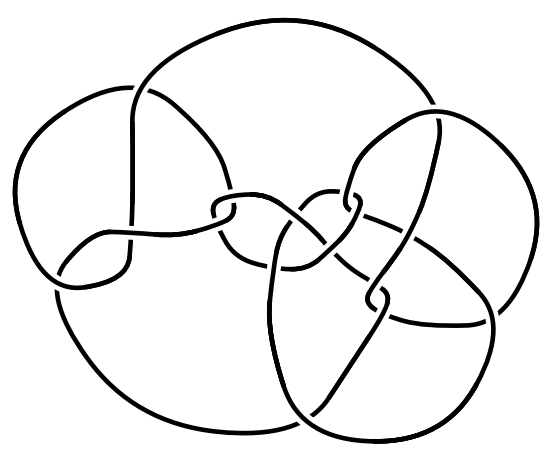

Figure 2: Braid positive knot $16_{472381}$

Example 15 Consider the 16 crossing homogeneous knot $K=16_{472381}$ [6] in Figure 2. This knot has braid index 4. No 4-braids with closure $K$ are homogeneous, and moreover all have length at least 17 , which implies $\ell_{K}(4)=17$. Computations of Stoimenow [11, Example 7] show that there is a homogeneous 5-braid representing $K$ with length 16 . So, $\ell_{K}(5)=16$. Now applying Theorem 8 , it follows that $\ell_{K}(n)=$ 
$n+11$ for all $n \geq 5$. Therefore the function drops once from $\ell_{K}(4)$ to $\ell_{K}(5)$ and then remains stable thereafter.

Example 16 Let $T_{p, q}$ denote the $(p, q)$-torus knot, where $p<q$. Torus knots are homogeneous knots, since they have a braid representative with $p$ strands and $q$ positive half twists (in fact, torus knots are braid positive knots). Recall that $T_{p, q}$ has the following invariants $[7 ; 8]$ :

$$
\begin{aligned}
& \operatorname{br}\left(T_{p, q}\right)=p \\
& g\left(T_{p, q}\right)=\frac{(p-1)(q-1)}{2}
\end{aligned}
$$

Applying Theorem 8,

$$
\ell_{T_{p, q}}(n)=n+(p-1)(q-1)-1
$$

for all $n \geq p$. This completes the computation of $\ell_{T_{p, q}}(n)$ for any $(p, q)$-torus knot.

Example 17 Let $K$ denote the figure eight knot. $K$ can be represented by the homogeneous 3-braid $\beta=\sigma_{1} \sigma_{2}^{-1} \sigma_{1} \sigma_{2}^{-1}$ (Figure 3). By Theorem 8,

$$
\ell_{K}(n)=n+2 g(K)-1=n+1
$$

for all $n \geq 3$, since $g(K)=1$. As the figure eight knot has braid index 3, the function $\ell_{K}(n)$ is therefore stable on all of its domain.

Notice that in both Example 13 and Example 17, $\ell_{K}(n)=n+1$. Theorem 9 shows that these two knots, the trefoil and the figure eight, are the only knots for which this is the case.

Theorem 9 Let $K$ be a knot. Then $\ell_{K}(n)=n+1$ for some $n$ if and only if $K$ is the trefoil or the figure eight knot. Hence, $\ell_{K}(n) \geq n+3$ for all nontrivial knots $K$ which are not the trefoil or figure eight.

Proof From Example 13 and Example 17, the "if" direction of the argument is clear.

Conversely, let $K$ be a knot such that $\ell_{K}(n)=n+1$ for some $n$. Observe that $K$ must be nontrivial since $\ell_{\text {unknot }}(n)=n-1$ for all $n \geq 1$.

Since $\ell_{K}(n)=n+1$, there is an $n$-braid $\beta$ with $n+1$ crossings and closure $\hat{\beta}=K$. As in the proof of Theorem 5, consider the array of $n-1$ integers associated to $\beta$. Again, observe that none of the numbers in this array associated to $\beta$ are 0 , for then 
$\widehat{\beta}$ would be a split link. By the pigeonhole principle, the array must be in one of the following forms:

$$
(1, \ldots, 1,3,1 \ldots, 1) \quad \text { or } \quad(1, \ldots, 1,2, \underbrace{1, \ldots, 1}_{r}, 2,1, \ldots, 1) .
$$

If the array of integers associated to $\beta$ is $(1, \ldots, 1,3,1, \ldots, 1)$, then by conjugating and destabilizing the braid, we get a Markov equivalent 2-braid $\beta^{\prime}$ with 3 crossings, which implies that $K$ is isotopic to the trefoil.

On the other hand, suppose the array is the latter of the two. If $r>0$, then $K$ must be a three component link, contradicting the assumption that $K$ is a knot. So the array must be of the form $(1, \ldots, 1,2,2,1, \ldots, 1)$. By conjugating and destabilizing, we get a Markov equivalent braid with associated array $(2,2)$. So $K$ can be expressed as a 3 -braid with 4 crossings. The only nontrivial knots which have diagrams with four crossings are the trefoil and the figure eight. Therefore, $K$ must be either the trefoil or the figure eight.

Thus $\ell_{K}(n)>n+1$ for all $n$, where $K$ is a nontrivial knot which is not the trefoil or figure eight. By part 2 of Theorem $4, \ell_{K}(n)-n$ is odd, so $\ell_{K}(n) \geq n+3$.

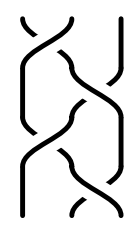

Figure 3: A homogeneous braid with closure the figure eight knot

More generally, we have the following result.

Theorem 10 Let $a$ be an odd integer. There are only finitely many prime knots $K$ with stable function $\ell_{K}(n)=n+a$.

Proof To prove this result, it suffices to show that if $K$ has stable function $\ell_{K}(n)=$ $n+a$, then $K$ has a braid representative $\beta \in B_{a+2}$ with $2(a+1)$ crossings (because only finitely many such braids exist).

Suppose that $\ell_{K}(n)=n+a$ for all $n \geq n_{0}$. Let $N \geq n_{0}$. Then $\ell_{K}(N)=N+a$. We can then find a braid $\beta^{\prime} \in B_{N}$ with closure $K$ and $N+a$ crossings. As before, by the pigeonhole principle $\beta^{\prime}$ can be destabilized $N-a-2$ times, yielding an $(a+2)$-braid $\beta$ with $2 a+2$ crossings and closure $K$, as desired. 
The last question about stability we investigate here is: For which knots is the function $\ell_{K}(n)$ stable on all of its domain? Observe that functions which are stable on their entire domain are determined by the braid index and braid length of $K$ :

$$
\ell_{K}(n)=\ell(K)+n-\operatorname{br}(K)
$$

for all $n \geq \operatorname{br}(K)$. Certainly, if there exists a braid $\beta \in B_{n}$ with $\ell(K)$ crossings and closure $K$ where $n>\operatorname{br}(K)$ then $\ell_{K}(n)$ is not stable on its domain. The knot $10_{136}$ is the first example of this. On the other hand, we can find many conditions under which the function is stable everywhere. The following theorem assimilates some sufficient conditions, primarily by applying the results of this section.

Theorem 11 Let $K$ be a knot. Let $\beta \in B_{\mathrm{br}(K)}$ be a braid of length $\ell(K)$ with closure $K$, where $\operatorname{br}(K)$ is the braid index of $K$.

(1) If $\ell(K)<2 \operatorname{br}(K)+2$, then $\ell_{K}(n)$ is stable on its entire domain.

(2) If $\ell(K)=\operatorname{br}(K)+2 g(K)-1$, then $\ell_{K}(n)$ is stable on its entire domain.

Proof of (1) To simplify notation, let $m=\operatorname{br}(K)$. Notice that by hypothesis, $\ell_{K}(m)=$ $\ell(K)$. We consider two cases.

If $\ell(K)=2 m+1$, part 2 of Theorem 5 implies that $\ell_{K}(n)=\ell_{K}(m+1)+n-(m+1)$ for all $n \geq m+1$. But since $\ell_{K}(m)=\ell(K)$, we can conclude by part 2 of Theorem 4 that $\ell_{K}(m+1)=\ell_{K}(m)+1=\ell(K)+1$. Therefore $\ell_{K}(n)=\ell(K)+n-m$ for all $n \geq m$. So $\ell_{K}(n)$ is stable on its entire domain.

If $\ell(K) \leq 2 m$, Theorem 5 part 1 implies that $\ell_{K}(n)=\ell_{K}(m)+n-m$ for all $n \geq m$, so the function is stable on its entire domain.

Proof of (2) Letting $m=\operatorname{br}(K)$, we have $\ell_{K}(m)=\ell(K)=m+2 g(K)-1$. It follows from parts 1 and 3 of Theorem 4 that $\ell_{K}(n)=n+2 g(K)-1$ for all $n \geq m$. Therefore the function is stable on its entire domain.

\section{Evaluating $\ell_{K}(n)$ for knots with low crossing number}

The results of Theorem 11 suffice to show that the function $\ell_{K}(n)$ is stable on its entire domain for all except four knots with eight or fewer crossings. The only outstanding knots are $7_{3}, 7_{5}, 8_{20}$, and $8_{21}$. We can show that these four knots also have functions that are stable everywhere by doing a little more work as follows.

First, we observe that these four knots have several identical knot invariants. For $K \in\left\{7_{3}, 7_{5}, 8_{20}, 8_{21}\right\}$, we have $\operatorname{br}(K)=3, \ell(K)=8, g(K)=2, \ell_{K}(3)=8$, and 
$\ell_{K}(4)=9$. By Theorem $5, \ell_{K}(5)$ determines $\ell_{K}(n)$ for all $n \geq 5$. So it remains to compute $\ell_{K}(5)$. We know $\ell_{K}(5)$ is an even integer and has the following bounds: $8 \leq \ell_{K}(5) \leq 10$. Therefore, it suffices to show that $\ell_{K}(5) \neq 8$.

Suppose that $\ell_{K}(5)=8$. Then there exists a braid $\beta \in B_{5}$ of length 8 with closure $K$. Using an analysis similar to the proof of Theorem 5, it follows that the array of four integers associated to $\beta$ is $(2,2,2,2)$.

For a moment, consider the "shadow" of the braid $\beta$ (that is, disregard whether crossings are over or under, writing the corresponding braid generators without exponents \pm 1 ). Then each $\sigma_{i}$ occurs exactly two times for all $i \in\{1,2,3,4\}$. Scanning through the braid word $\beta$, consider the cyclic order in which consecutive generators $\sigma_{i}$ and $\sigma_{i+1}$ occur in the word. Up to cyclic permutation, the elements appear as

$$
\sigma_{i} \cdots \sigma_{i+1} \cdots \sigma_{i} \cdots \sigma_{i+1} \cdots \quad \text { or } \quad \sigma_{i} \cdots \sigma_{i} \cdots \sigma_{i+1} \cdots \sigma_{i+1} \cdots \text {. }
$$

If any two consecutive generators occur in this second configuration listed above, the braid shadow must (up to conjugation) look like Figure 4. The figure pictures the case where $i=2$. The boxes in the figure may be filled in with any braid so that the condition $\ell_{K}(5)=8$ is satisfied. The case where $i=1$ has a similar figure, with the leftmost strand missing and another strand added on the right hand side (similarly for $i=3)$.

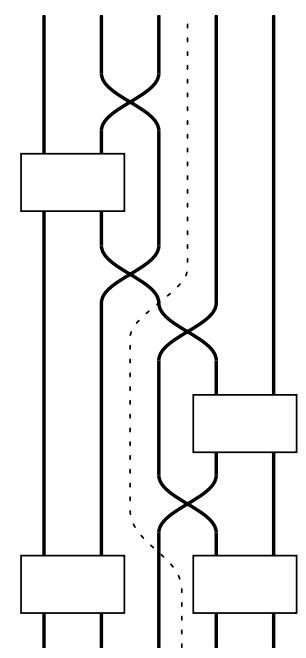

Figure 4: Possible braid shadow for a braid satisfying $b_{5}(K)=8$. The dotted line points out that the closed braid will be a connected sum. 
Notice that if two consecutive generators appear in this order, the closed braid splits as a connected sum. This is due to the existence of the dotted line in Figure 4 which meets the braid in only two points. Since $K$ is a prime knot of 7 or 8 crossings, this implies a contradiction. Therefore, the braid generators must occur in the cyclic ordering $\sigma_{i} \cdots \sigma_{i+1} \cdots \sigma_{i} \cdots \sigma_{i+1} \cdots$ for $i \in\{1,2,3\}$. It then follows that the only possible braid shadow (up to braid relations) for $\beta$ is $\sigma_{1} \sigma_{2} \sigma_{3} \sigma_{4} \sigma_{1} \sigma_{2} \sigma_{3} \sigma_{4}$ (or equivalently, $\left.\sigma_{1} \sigma_{2} \sigma_{1} \sigma_{3} \sigma_{2} \sigma_{4} \sigma_{3} \sigma_{4}\right)$ as in Figure 5 .

A priori, the braid $\beta$ could still be one of $2^{8}$ different braids since there are eight crossings in the braid shadow, each of which must be assigned to be either an overcrossing or an undercrossing. However, several choices of crossings can be ruled out. First of all, the $\sigma_{1}$ 's must have the same exponent signs, for otherwise a braid relation would allow a sequence of destabilizations and conjugations resulting in a braid $\beta^{\prime}$ with 4 strands and 7 crossings, contradicting $\ell_{K}(4)=9$. In addition, the first $\sigma_{2}$ in the braid word must have a different exponent sign than the $\sigma_{1}$ 's for the same reasons. By symmetry, these arguments also apply to the $\sigma_{3}$ 's and the $\sigma_{4}$ 's. In summary, the braid $\beta$ must be of the form:

$$
\sigma_{1} \sigma_{2}^{-1} \sigma_{1} \sigma_{3}^{ \pm 1} \sigma_{2}^{ \pm 1} \sigma_{4} \sigma_{3}^{-1} \sigma_{4} \quad \text { or } \quad \sigma_{1} \sigma_{2}^{-1} \sigma_{1} \sigma_{3}^{ \pm 1} \sigma_{2}^{ \pm 1} \sigma_{4}^{-1} \sigma_{3} \sigma_{4}^{-1} .
$$

(Mirror images of braids yield the same knot.) Thus there are only 8 different possibilities for $\beta$. The closures of these eight braids are: the unknot, $3_{1}, 6_{1}, 7_{7}$, and 812 .

So none of the four knots $7_{3}, 7_{5}, 8_{20}, 8_{21}$ can be formed by closing a braid whose associated array of integers is $(2,2,2,2)$. This implies $\ell_{K}(5) \neq 8$, and therefore $\ell_{K}(5)=10$, where $K \in\left\{7_{3}, 7_{5}, 8_{20}, 8_{21}\right\}$. Now applying Theorem 5 , it follows that $\ell_{K}(n)=n+5$ for all $n \geq 3$, where $K \in\left\{7_{3}, 7_{5}, 8_{20}, 8_{21}\right\}$. So the function $\ell_{K}(n)$ is stable on its entire domain for all knots $K$ with eight or fewer crossings.

Nine crossing knots present a bigger challenge in terms of computing $\ell_{K}(n)$. The results of Theorem 11 are sufficient for showing $\ell_{K}(n)$ is stable on its entire domain for all except nineteen of the nine crossing knots.

For these nineteen knots, we can show that the functions are again stable everywhere, but as with the four knots mentioned earlier, a bit more work is required.

For all nine crossing knots $K$ except $9_{35}$ we need only rule out the possibility that there is a 7-braid with 12 crossings and closure $K$ to show that $\ell_{K}(n)$ is stable on its entire domain. Using a computer program to check all 12-crossing braids, this possibility was eliminated, forcing us to conclude that all of these nine crossing knots have stable $\ell_{K}(n)$ functions. Similarly, $\ell_{9_{35}}(n)$ can be shown to be stable everywhere using computation. 


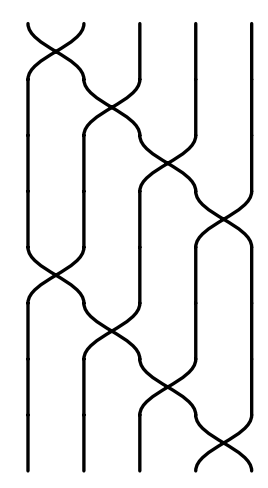

Figure 5: Braid shadow for a braid satisfying $b_{5}(K)=8$

Hence for any knot $K$ with nine or fewer crossings, braid length cannot be decreased by increasing the number of braid strands. In view of this discussion, we have the following theorem:

Theorem 12 Let $K$ be a knot with nine or fewer crossings. Then $\ell_{K}(n)$ is stable on its entire domain.

The first example of a knot whose function is not stable everywhere is $10_{136}$, which was analyzed in the introduction. Other knots without stable functions include: $11 \mathrm{n}_{8}$, $11 \mathrm{n}_{121}, 11 \mathrm{n}_{131}, 12 \mathrm{n}_{20}, 12 \mathrm{n}_{24}, 12 \mathrm{n}_{65}, 12 \mathrm{n}_{119}, 12 \mathrm{n}_{358}, 12 \mathrm{n}_{362}, 12 \mathrm{n}_{403}, 16_{472381}$, and $16_{1223549}$ [4; 11]. In each of these examples, the function drops one time, and then is stable thereafter. There are no known examples of a function that drops more than once.

\section{References}

[1] J Alexander, A lemma on systems of knotted curves, Proc. Nat. Acad. Sci. USA 9 (1923) 93-95

[2] J S Birman, Braids, links, and mapping class groups, Annals of Mathematics Studies 82, Princeton University Press, Princeton, N.J. (1974) MR0375281

[3] J S Birman, T E Brendle, Braids: a survey, from: "Handbook of knot theory", Elsevier B. V., Amsterdam (2005) 19-103 MR2179260

[4] J Cha, C Livingston, KnotInfo: an online table of knot invariants Available at http://www.indiana.edu/ knotinfo/ 
[5] T Gittings, Minimum braids: A complete invariant of knots and links arXiv: math.GT/0401051

[6] J Hoste, M Thistlethwaite, KnotScape Available at http://www.math.utk.edu/ $\sim$ morwen/knotscape.html

[7] V F R Jones, Hecke algebra representations of braid groups and link polynomials, Ann. of Math. (2) 126 (1987) 335-388 MR908150

[8] K Murasugi, On the braid index of alternating links, Trans. Amer. Math. Soc. 326 (1991) 237-260 MR1000333

[9] K Murasugi, B I Kurpita, A study of braids, Mathematics and its Applications 484, Kluwer Academic Publishers, Dordrecht (1999) MR1731872

[10] J R Stallings, Constructions of fibred knots and links, from: "Algebraic and geometric topology (Proc. Sympos. Pure Math., Stanford Univ., Stanford, CA, 1976), Part 2", Proc. Sympos. Pure Math., XXXII, Amer. Math. Soc., Providence, R.I. (1978) 55-60 MR520522

[11] A Stoimenow, On the crossing number of positive knots and braids and braid index criteria of Jones and Morton-Williams-Franks, Trans. Amer. Math. Soc. 354 (2002) 3927-3954 MR1926860

Department of Mathematics, Indiana University

Bloomington IN 47405, USA

cvancott@indiana.edu

http://mypage.iu.edu/ cvancott/

Received: 14 August 2006 Revised: 8 December 2006 